\title{
Cambios en la Bioquímica Sérica en Crías de Alpaca con Diarrea
}

\author{
Changes in the Serum Biochemistry in Baby Alpacas with Diarrhea
}

José Rodríguez G. ${ }^{1,5}$, Manuel Barrios-Arpi², María Vásquez C. ${ }^{3}$, Boris Lira M. ${ }^{3}$, Siever Morales C. ${ }^{4}$, Juan Lucas L. ${ }^{1}$, Bernardo López-Torres ${ }^{1}$

\section{Resumen}

El objetivo del presente estudio fue determinar las alteraciones que ocurren en los parámetros bioquímicos sanguíneos en crías de alpaca que cursan con diarrea infecciosa. Se utilizaron 80 animales de las regiones Pasco y Junín, Perú. Los animales fueron distribuidos de acuerdo a su edad: menores de 1 mes de edad (10 controles y 40 enfermos) y 1-2 meses de edad (10 controles y 20 enfermos). La diarrea clínica tenía 2-3 días de presentación en el momento de la toma de muestras. Se hicieron determinaciones de glucosa, triglicéridos, colesterol, proteína total, albúmina, calcio, bilirrubina total, bilirrubina directa y fosfatasa alcalina. Los parámetros bioquímicos, excepto glucosa, cambiaron significativamente en los animales enfermos con respecto a los controles. En los animales enfermos, los niveles de proteína total, albúmina y calcio disminuyeron significativamente $(\mathrm{p}<0.05)$, mientras que los niveles de fosfatasa alcalina, bilirrubina, colesterol y triglicéridos se incrementaron significativamente $(\mathrm{p}<0.05)$.

Palabras clave: bioquímica sanguínea; crías de alpaca; diarrea

\section{AbSTRaCT}

The aim of this study was to determine alterations in blood biochemistry parameters in baby alpacas with infectious diarrhea. Eighty alpacas from Junin and Pasco regions (Peru) were used in the study. The animals were grouped by age in: less than 1 month old

${ }^{1}$ Centro Experimental IVITA-El Mantaro, Universidad Nacional Mayor de San Marcos, Huancayo, Perú

${ }^{2}$ Laboratorio de Patología Clínica y Biología Molecular, ${ }^{3}$ Laboratorio de Fisiología Animal, ${ }^{4}$ Laboratorio de Microbiología y Parasitología Veterinaria, Facultad de Medicina Veterinaria, Universidad Nacional Mayor de San Marcos, Lima, Perú

${ }^{5}$ E-mail: jrodriguezg3@unmsm.edu.pe

Trabajo ejecutado con fondos provenientes del Proyecto N. ${ }^{\circ}$ 173- FINCyT-IB-2013, Lima, Perú

Recibido: 18 de diciembre de 2016

Aceptado para publicación: 21 de abril de 2017 
(10 control and 40 with diarrhea) and 1-2 months old (10 control and 20 with diarrhea). Clinical diarrhea had 2-3 days of presentation at the time of sampling. Determinations of glucose, triglycerides, cholesterol, total protein, albumin, calcium, total bilirubin, direct bilirubin and alkaline phosphatase were made. Biochemical parameters, except glucose, significantly changed in diarrheic animals with respect to controls. In the animals with diarrhea the total protein, albumin and calcium levels were significantly decreased $(p<0.05)$, while the alkaline phosphatase, bilirubin, cholesterol and triglycerides were significantly increased $(\mathrm{p}<0.05)$.

Key words: blood biochemistry; baby alpaca; diarrhea

\section{INTRODUCCIÓN}

Las alpacas son los camélidos domésticos de mayor importancia en la economía de los Andes del Perú. La crianza de estos animales se da en las extensas áreas de pastos naturales en las zonas alto andinas, donde no es posible la agricultura ni la crianza exitosa de otras especies domésticas (Iñiguez y Alem, 1996).

Las alpacas son susceptibles a un gran número de enfermedades infecciosas, especialmente en la etapa posnatal, que se traduce en grandes pérdidas económicas para el productor (FAO, 2005). Los problemas entéricos tienen un origen que frecuentemente es de carácter multifactorial. Los principales agentes infecciosos involucrados en la diarrea neonatal de alpacas son Clostridium perfringes tipo A y C, Escherichia coli, rotavirus, coronavirus y Cryptosporidium $\mathrm{sp}$ (Holland, 1990). Así mismo, la alta prevalencia de diarrea neonatal puede estar influenciada por factores no-infecciosos como trastornos metabólicos, incluyendo fallas en los procesos absortivos de carbohidratos a nivel intestinal, que pueden ocasionar la muerte del animal (Sklan, 2001; Ogra y Welliver, 2008).

Para un mejor entendimiento de los posibles factores involucrados en la diarrea neonatal es necesario conocer el desarrollo fisiológico del sistema digestivo de las crías de alpacas. Diversos estudios sobre el siste- ma gastrointestinal de estos animales demuestran que las células intestinales, los receptores proteicos de absorción, las células y los órganos exocrinos y endocrinos (anexos gastrointestinales) alcanzan su madurez a medida que las crías alcanzan los 45 días de edad, aproximadamente, que es cuando presenta un sistema digestivo suficientemente desarrollado e inmunológicamente maduro, capaz de resistir los trastornos infecciosos y no-infecciosos (Lira et al., 2012; Rodríguez et al., 2012, 2015a; Hidalgo et al., 2015).

La diarrea neonatal infecciosa y no infecciosa en crías de alpaca merma la salud del animal, lo que se refleja en alteraciones en los parámetros fisiológicos (Rodríguez et al., 2015b). Además, afecta la capacidad digestiva y de absorción intestinal en estos animales, de allí que el presente estudio tuvo por objetivo determinar los cambios de los parámetros bioquímicos sanguíneos (glucosa, triglicéridos, colesterol, proteína total, albúmina, calcio, bilirrubina total, bilirrubina directa y fosfatasa alcalina) en crías de alpaca de dos grupos etarios que presentaban diarrea neonatal positiva a colibacilosis.

\section{Materiales Y MéTodos}

\section{Lugar de Ejecución}

El estudio se realizó en las comunidades alpaqueras de las regiones Junín y Pasco (Perú), entre enero y marzo de 2014 y 2015. 
Las zonas se encuentran ubicadas por encima de los $3500 \mathrm{msnm}$. Las muestras de suero fueron procesadas en la estación experimental del Centro de Investigación IVITA, sede El Mantaro, de la Facultad de Medicina Veterinaria, Universidad Nacional Mayor de San Marcos.

\section{Animales y Muestras}

Se utilizaron 80 crías de alpacas: 20 clínicamente sanas (controles) y 60 con diarreas de tipo infeccioso, positivas a colibacilosis, determinado por aislamiento microbiológico convencional (MINSA, 2005; OIE, 2008). Los animales fueron agrupados en dos grupos etarios: menores de 1 mes de edad ( 10 controles y 40 enfermos), y entre 1-2 meses de edad (10 controles y 20 enfermos).

Se tomaron muestras de sangre por punción de la vena yugular entre los 2-3 días de la presentación de diarrea. La sangre fue colectada en tubos de ensayo $\sin$ anticoagulante y centrifugada a $3000 \mathrm{~g}$ por 30 min en una centrífuga automática (Gemmy, Taiwán). El suero resultante se almacenó a $-196{ }^{\circ} \mathrm{C}$ en un tanque de nitrógeno líquido (Gemmy, Taiwán).

El manejo de los animales se realizó siguiendo los «Principios Directrices Internacionales para la Investigación Biomédica que implique el Uso de Animales» del Consejo de Organizaciones Internacionales de las Ciencias Médicas (CIOMS, 1985).

\section{Análisis Bioquímico Sanguíneo}

Mediante análisis bioquímico del suero sanguíneo se determinaron los niveles de glucosa $(\mathrm{G}, \mathrm{mg} / \mathrm{dl})$, triglicéridos $(\mathrm{T}, \mathrm{mg} / \mathrm{dl})$, colesterol $(\mathrm{Co}, \mathrm{mg} / \mathrm{dl})$, proteína total $(\mathrm{P}, \mathrm{g} / \mathrm{dl})$, albúmina $(\mathrm{A}, \mathrm{g} / \mathrm{dl})$, calcio $(\mathrm{Ca}, \mathrm{mg} / \mathrm{dl})$, bilirrubina total (BT, $\mathrm{mg} / \mathrm{dl}$ ), bilirrubina directa $(\mathrm{BD}, \mathrm{mg} / \mathrm{dl})$ y fosfatasa alcalina $(\mathrm{FA}, \mathrm{U} /$ I). Se utilizaron kits comerciales (FAR Diagnostics, Italia) y se siguieron las especi- ficaciones del fabricante. La lectura se hizo en un analizador bioquímico semiautomático (Sinowa, China).

\section{Análisis de Datos}

Los niveles de los parámetros bioquímicos sanguíneos se presentan como promedios y desviaciones estándares. Para determinar las diferencias significativas entre los grupos control y enfermo se usó el análisis de varianza con la prueba poshoc de Dunnett, con un nivel de significación a partir de1 95\%. Para determinar la significancia de los parámetros bioquímicos entre el total de crías controles (C) y total de crías enfermas (E), se realizó la prueba de t-Student a partir del $95 \%$ de nivel de confianza.

\section{Resultados}

En el Cuadro 1 se observa que el total de animales con diarrea presentaban cambios significativos con respecto al total de animales controles en todos los parámetros bioquímicos sanguíneos, excepto para glucosa. Se produjo una disminución significativa $(\mathrm{p}<0.001)$ de los niveles de P, A y Ca, y un incremento significativo de los niveles de $\mathrm{T}$ $(p<0.01)$ y Co, BD, BT y FA $(p<0.001$; Figura 1$)$.

\section{Discusión}

En términos generales, se podría decir que la diarrea es la alteración del movimiento de iones y agua que siguen un gradiente osmótico a través de la pared intestinal. Bajo condiciones normales, el tracto gastrointestinal tiene la capacidad para poder movilizar grandes cantidades de agua y electrolitos desde el lumen intestinal hacia sangre y viceversa. El movimiento alterado puede ocurrir, ya sea por trasportadores o por espacios intercelulares, los que son regulados por las uniones estrechas. 
Cuadro 1. Valores bioquímicos séricos del total de crías de alpaca sin diarrea (controles) y total de crías con diarrea infecciosa positiva a colibacilosis

\begin{tabular}{ccc}
\hline Variable $^{1}$ & Control & Con diarrea \\
\hline P & $7.3 \pm 0.5^{\mathrm{a}}$ & $5.8 \pm 0.4^{\mathrm{b}, 1}$ \\
$\mathrm{~A}$ & $5.4 \pm 0.3^{\mathrm{a}}$ & $4.2 \pm 0.6^{\mathrm{b}, 1}$ \\
$\mathrm{Co}$ & $57 \pm 7.5^{\mathrm{a}}$ & $110 \pm 15^{\mathrm{b}, 1}$ \\
$\mathrm{~T}$ & $54 \pm 9^{\mathrm{a}}$ & $88 \pm 17^{\mathrm{b}, 2}$ \\
$\mathrm{G}$ & $185 \pm 15^{\mathrm{a}}$ & $172 \pm 17^{\mathrm{a}}$ \\
Ca & $13 \pm 1.2^{\mathrm{a}}$ & $8.5 \pm 0.6^{\mathrm{b}, 1}$ \\
BD & $0.35 \pm 0.2^{\mathrm{a}}$ & $1.8 \pm 0.06^{\mathrm{b}, 1}$ \\
BT & $0.3 \pm 0.08^{\mathrm{a}}$ & $2.6 \pm 0.06^{\mathrm{b}, 1}$ \\
FA & $60 \pm 4.9^{\mathrm{a}}$ & $172 \pm 13^{\mathrm{b}, 1}$ \\
\hline
\end{tabular}

${ }^{1} \mathrm{G}$ : glucosa (mg/dl); T: triglicéridos ( $\left.\mathrm{mg} / \mathrm{dl}\right)$; Co: colesterol $(\mathrm{mg} / \mathrm{dl}) ; \mathrm{P}$ : proteína total $(\mathrm{g} / \mathrm{dl}) ; \mathrm{A}$ : albúmina (g/dl); Ca: calcio $(\mathrm{mg} / \mathrm{dl}) ; \quad \mathrm{BT}$ : bilirrubina total (mg/dl); BD: bilirrubina directa (mg/dl); FA: fosfatasa alcalina (U/l)

a,b Superíndices diferentes entre tratamientos indican diferencia estadística (1: $p<0.01 ; 2$ : $p<0.001$ )

Entre los transportadores que podrían verse alterados se encuentra el SGLT-1, presente en alpacas (Rodríguez et al., 2012), intercambiador $\mathrm{Na}^{+} / \mathrm{H}^{+}$y las tres isoformas del intercambiador $\mathrm{Cl}^{-} / \mathrm{HCO}_{3}$ en la zona apical celular. Por otra parte, los patógenos entéricos, como E. coli, pueden alterar directamente los procesos de transporte iónico o hacerlo indirectamente a través de la inflamación, neuropéptidos o por la pérdida de superficie de absorción. Es conocido que E. coli Enterohemorrágica (EHEC) causa pérdida de las microvellosidades, lo que disminuye el área de superficie para la absorción de nutrientes y ocasiona un aumento de la osmolaridad del contenido intestinal y malabsorción (Nataro y Kaper, 1998).

Las crías de alpaca con diarrea y menores del mes de edad fueron las que mostraron menores niveles de $\mathrm{P}$, A y Ca con res- pecto a las crías con diarrea de 1-2 meses de edad y a los controles de ambas edades. Durante los procesos entéricos infecciosos que cursan con diarrea hay dificultades en la digestión y absorción de los nutrientes como proteínas e iones como el calcio, lo que afecta el paso de estos elementos a sangre, disminuyendo sus niveles sanguíneos (Gyles et al., 2010; Tajik y Nazifi, 2013). Por otra parte, la falta de apetito, en los procesos entéricos con diarrea, contribuirá a la disminución de los niveles sanguíneos de proteínas, albúmina y calcio (Gyles et al., 2010).

Además, los menores niveles de $\mathrm{P}, \mathrm{A}$ y Ca podrían estar relacionados a una menor absorción de nutrientes que depende del área intestinal, cuyo desarrollo y madurez es dependiente de la edad. Se ha demostrado que a los 45 días de edad se presenta un mayor desarrollo intestinal con respecto a edades más tempranas (Lira et al., 2012; Rodríguez et al., 2012, 2015a; Hidalgo et al., 2015). El menor desarrollo intestinal en crías menores de un mes de edad las hace más susceptibles a los efectos infecciosos de patógenos entéricos como E. coli; en cambio, los animales de mayor edad son más eficientes en digerir y absorber los nutrientes proteicos y iones como el calcio, así como un sistema digestivo más desarrollado con una mejor barrera de defensa inmune ante posibles infecciones por patógenos causantes de diarrea (Tajik y Nazifi, 2013; Hashemnia et al., 2014; Singh et al., 2014).

En contraparte al presente estudio, se ha reportado en terneros con diarrea que sus niveles de proteína total aumentan significativamente con respecto a animales sanos, mientras que la albúmina presenta niveles similares entre animales con y sin diarrea (Tajik y Nazifi, 2013); asimismo, otros electrolitos distintos al calcio no se incrementaron en terneros con diarrea a excepción del potasio (Seifi et al., 2006). Por otra parte, Pekcan et al. (2012) no encontraron diferencias en los niveles de proteína total y albúmina entre terneros sanos y con diarrea. 

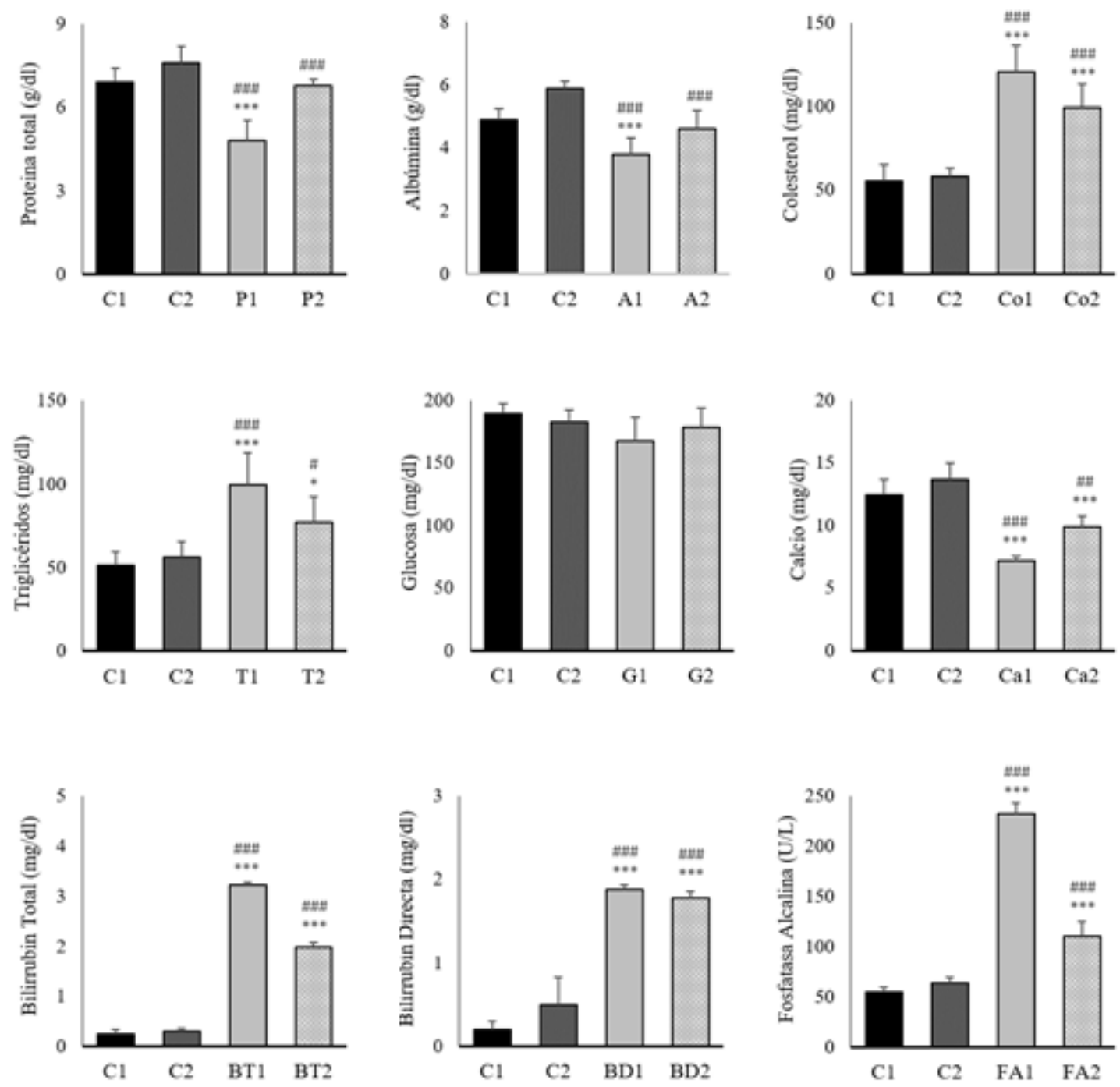

Figura 1. Parámetros bioquímicos sanguíneos en crías de alpacas control (sin diarrea) menores de 1 mes de edad (C1) y de 1-2 meses de edad (C2) y crías con cuadros de diarrea infecciosa menores de 1 mes de edad (PT1, A1, Co1, T1, G1, Ca1, BD1, BT1 y FA1) y de 1-2 meses de edad (PT2, A2, Co2, T2, G2, Ca2, BD2, BT2 y FA2). Los datos se expresan como promedio y desviación estándar. Los valores de las crías con diarrea se compararon con ambos controles: $\mathrm{C} 1\left(99.9 \%^{* * *}\right.$ y $\left.95 \%{ }^{*}\right)$ y C2 $\left(99.9 \%{ }^{\# \# \#}\right.$ y $\left.95 \%^{*}\right)$

Los niveles de BD y BT aumentaron en las crías de alpaca con cuadros de diarrea (Cuadro 1), especialmente en las crías con diarrea menores al mes de edad (Figura 1). El aumento de bilirrubina en animales domésticos puede, en ocasiones, ser debido a causas ajenas a enfermedades hepáticas, tales como enfermedades hemolíticas con incremento mayor de la bilirrubina no conjugada en los primeros días (Benjamín, 1991). Sin embargo, se sabe que E. coli estaría involucrado en la formación de abscesos en hígado con infiltración de neutrófilos y células mononucleares en ovinos, bovinos, búfalos y camellos (Scanlan y Edwards, 1990; Mohamed et al., 1997; Lechtenberg et al., 1998; Nagaraja y Chengappa, 1998; Sayed et al., 2008), lo cual podría contribuir al in- 
cremento de los niveles de bilirrubina directa por obstrucción de los canalículos biliares, incrementando los niveles de bilirrubina total.

Los valores de fosfatasa alcalina se encontraron incrementados en ambos grupos, siendo mayores en crías menores de 1 mes de edad. Esto último es debido a que, como sucede en otras especies, los niveles de esta enzima se elevan en animales en crecimiento debido al desarrollo óseo y su relación con la marcada actividad enzimática encontrada en los osteoblastos (Kaneko et al., 2008). Los niveles mayores en crías con diarrea coinciden con otros autores, donde la actividad total de fosfatasa alcalina se vio incrementada, muy posiblemente, por lesiones histopatológicas en el hígado, lo cual refleja una alteración funcional de este órgano (Lechowski,1996).

Los niveles de colesterol resultaron disminuidos en las crías con diarrea en proporciones similares para los dos grupos de animales afectados, coincidiendo con Pekcan et al. (2012), donde los niveles de colesterol se encontraron dos veces por encima de los valores hallados para animales sin diarrea.

Finalmente, los valores encontrados para triglicéridos se incrementaron en las crías con diarrea, especialmente en el grupo de crías de menos de 1 mes de edad. No se dispone de reportes de cambios en niveles de triglicéridos por efecto de la diarrea en crías de animales, pero se sabe que $E$. coli estaría involucrado, alterando posiblemente el metabolismo de las grasas (Sayed et al., 2008).

\section{Conclusiones}

- Los valores de proteínas totales, albúmina y calcio se encuentran disminuidos, en tanto que los valores de fosfatasa alcalina, bilirrubina, triglicéridos y colesterol se encuentran incrementados en crías de alpaca con diarrea de alpaca con diarrea infecciosa.
- Los valores de glucosa se mantienen normales en crías de alpaca con diarrea infecciosa.

\section{Literatura Citada}

1. Benjamín M. 1991. Manual de patología clínica veterinaria. México: Ed Limusa. $421 \mathrm{p}$.

2. [FAO] Food and Agriculture Organization of the United Nations.

2005. Situación actual de los camélidos sudamericanos en Perú. Proyecto de cooperación técnica en apoyo a la crianza y aprovechamiento de los camélidos sudamericanos en la región andina. Lima: FAO. $63 \mathrm{p}$.

3. Gyles CL, Prescott JF, Songer JG, Thoen CO. 2010. Pathogenesis of bacterial infections in animals. $4^{\text {th }}$ ed. Iowa: Wiley-Blackwell. $664 \mathrm{p}$.

4. Hashemnia M, Khodakaram-Tafti A, Mostafa S, Nazifi S. 2014. Hematological and serum biochemical analyses in experimental caprine coccidiosis. J Parasit Dis 38: 116-123. doi: 10.1007/s12639-012-0205-1

5. Hidalgo C, Vásquez M, Lira B, Rodríguez J. 2015. Identificación de células enteroendocrinas productoras de péptido similar al glucagón tipo 1 (GLP1) en el intestino de la alpaca. Rev Inv Vet Perú 26: 373-380. doi: 10.15381/ rivep.v26i3.11172

6. Holland R. 1990. Some infectious causes of diarrhea in young farm animals. Clin Microbiol Rev 3: 345-375.

7. Iñiguez LC, Alem R. 1996. Role of camelids as means of transportation and exchange in the Andean region of Bolivia. World Anim Rev 86: 12-21.

8. [CIOMS] Council for International Organizations of Medical Sciences. 1985. International guiding principles for biomedical research involving animals. Geneva. [Internet]. Available in: http:// cioms.ch/publications/guidelines/ 1985_texts_of_guidelines.htm 
9. Kaneko JJ, Harvey JW, Bruss ML. 2008. Clinical biochemistry of domestic animals. $6^{\text {th }}$ ed. USA: Academic Press. $928 \mathrm{p}$.

10. Lechowski R. 1996. Changes in the profile of liver enzymes in newborn calves induced by experimental, subclinical acidosis in pregnant cows and osmotic diarrhoea. Vet Res Commun 20: 351-365. doi: 10.1007/BF00366542

11. Lechtenberg KF, Nagaraja TG, Leipold HW, Chengappa MM. 1988. Bacteriologic and histologic studies of hepatic abscesses in cattle. Am J Vet Res 49: 58-62.

12. Lira B, Cueva S, Rodríguez J, Ayón M, Zanuzzi C, Barbeito C, Falcón N, Vásquez M. 2012. Identificación de las células de Paneth en el intestino delgado de alpacas en los primeros 21 días de edad. Rev Inv Vet Perú 23: 138-146. doi: 10.15381/rivep.v23i2.893

13. [MINSA] Ministerio de Salud del Perú. 2005. Manual de procedimientos bacteriológicos en infecciones intrahospitalarias. Lima: CEPREDIM. Serie de Normas Técnicas N. ${ }^{\circ} 28.106 \mathrm{p}$.

14. Mohamed MH, El-Saied TI, Attia UH. 1997. Pathological studies on some liver affections in camels at Sharkia Governorate. Egypt J Comp Pathol Clinic Pathol 10(2): 17-27.

15. Nagaraja TG, Chengappa MM. 1998. Liver abscesses in feedlot cattle: a review. J Anim Sci 76: 287-298.

16. Nataro JP, Kaper JB. 1998. Diarrheagenic Escherichia coli. Clin Microbiol Rev 11: 142-201.

17. Ogra PL, Welliver RC Sr. 2008. Effects of early environment on mucosal immunologic homeostasis, subsequent immune responses and disease outcome. Nestle Nutr Workshop Ser Pediatr Program 61: 145-81. doi: 10.1159/ 0000113492

18. [OIE] Organización Mundial de Sanidad Animal. 2008. Salmonelosis. Cap. 2.9.9. En: Manual de las pruebas de diagnóstico y de las vacunas para los animales terrestres. $6^{\mathrm{a}}$ ed. Paris: OIE. p 1268-1286.
19. Pekcan M, Altintas A, Karagul H, Fidanci $U$, Uysal $H$, Besalti $O$, Unubol AS, et al. 2012. Serum biochemistry and native protein electrophoresis in diarrheic calves with arthritis. Acta Vet 62: 261-269. doi: 10.2298/AVB1203261P

20. Rodríguez J, Cueva S, Lira B, Espinoza J, Vásquez M. 2012. Identificación inmunohistoquímica de transportadores de glucosa intestinal y absorción de glucosa durante el desarrollo y maduración del intestino delgado de crías de alpacas. Rev Inv Vet Perú 23: 126137. doi: 10.15381/rivep.v23i2.892

21. Rodríguez J, Espinoza J, Rojas G, Lira $B$, Vásquez M, López-Torres B, Caro C. 2015a. Desarrollo de los Islotes de Langerhans en páncreas de alpacas neonatas. Rev Inv Vet Perú 26: 381-388. doi; 10.15381/rivep.v26i3.11171

22. Rodríguez J, Vásquez M, Lira B, Lucas J, Barrios-Arpi M, Morales S, López-Torres B. 2015b. Cambios en los parámetros fisiológicos por diarrea neonatal en crías de alpaca. Rev Complutense Cienc Vet 9(1): 1-9. doi: 10.5209/rev_RCCV.2015.v9.n1.47689

23. Sayed SM, Sayed GM, El-Nisr NA. 2008. Clinico-diagnostic studies on hepatic affections of aged buffaloes. Assiut Vet Med J 54: 310-328.

24. Scanlan CM, Edwards JF. 1990. Bacteriologic and pathologic studies of hepatic lesions in sheep. Am J Vet Res 51:363-366.

25. Seifi HA, Mohri M, Shoorei E, Farzaneh N. 2006. Using hematological and serum biochemical findings as prognostic indicators in calf diarrhoea. Comp Clin Pathol 15: 143-147. doi: 10.1007/s00580-006-0620-8

26. Singh RD, Gibbons SJ, Saravanaperumal SA, Du P, Henning GW, Eisenman ST, Mazzone A, et al. 2014. Ano1, a Ca2+-activated Cl- channel, coordinates contractility in mouse intestine by $\mathrm{Ca} 2+$ transient coordination between interstitial cells of Cajal. J Physiol 592(18): 4051-4068. doi: 10.1113/ jphysiol.2014.277152 
27. Sklan D. 2001. Development of the digestive tract of poultry. World Poultry Sci J 57: 415-428. doi: 10.1079/ WPS20010030
28. Tajik J, Nazifi S. 2013. A preliminary study of the correlations of serum concentrations of electrolytes and trace elements with clinical signs in diarrhoeic dairy calves. Pak Vet J 33: 5-8. 